\title{
NOTE ON KATO'S TYPE INEQUALITY
}

\section{FLAVIUS LUCIAN PATER}

Abstract. The main purpose of this note is to prove a Kato's type inequality for a generalized Schrödinger operator $\mathscr{A}$. As an application, we present the $L^{\infty}$-uniqueness of $\mathscr{A}$. Mathematics subject classification (2010): 35J10, $60 \mathrm{~J} 60$.

Keywords and phrases: Kato inequality, Schrödinger operator, uniqueness.

\section{REFERENCES}

[1] W. ARENDT, Kato's inequality: a characterization of generators of positive semigroups, Proc. Roy. Irish Acad. Sect. A, 84 (1984), 155-174.

[2] A. EBERLE, $L^{p}$ Uniqueness of non-symmetric diffusion operators with singular drift coefficients, J. Funct. Anal., 173 (2000), 328-342.

[3] T. KATO, Schrödinger operators with singular potentials, Israel J. Math., 13 (1972), 135-148.

[4] L. D. LemLe, $L^{\infty}\left(\mathbb{R}^{d}, d x\right)$-uniqueness of generalized Schrödinger operators, Differ. Geom.-Dyn. Syst., 9(2007), 103-110.

[5] L. D. LEMLE, $L^{1}\left(\mathbb{R}^{d}, d x\right)$-uniqueness of weak solution for the Fokker-Planck equation associated to a class of Dirichlet operators, Electron. Res. Announc. Math. Sci., 15(2008), 65-70.

[6] L. D. LEMLE, $L^{\infty}$-uniqueness of Schrödinger operators restricted in an open domain, in "Hot Topics in Operator Theory" (eds. Douglas, R. et al.), OT21 Conference Proceedings, Theta, Bucureşti, 2008, pp. 137-143.

[7] L. D. LEMLE, Existence and uniqueness for $C_{0}$-semigroups on the dual of a Banach space, Carpathian J. Math., 26(2010), 67-76.

[8] L. D. LemLe, L. M. Wu, Unicité des pré-générateurs dans les espaces localement convexes, C.R. Acad. Sci. Paris, 347(2009), 1281-1284.

[9] L. D. LemLe, L. M. WU, Uniqueness of $C_{0}$-semigroups on a general locally convex vector space and an application, Semigroup Forum, 82 (2011), 485-496.

[10] L. D. LEMLE, F. L. PATER, A. Berdie, Kato's type inequality for symmetric diffusion operators, in "Numerical Analysis and Applied Mathematics" (eds. Simos, T. et al.), AIP Conference Proceedings, vol.1389, Malville, New York, 2011, pp. 528-531.

[11] P. A. Meyer, W. A. Zheng, Construction du processus de Nelson reversible, Lect. Notes Math., vol. 1123, Springer, Berlin, 1984.

[12] M. RöcKNER, T. S. ZHANG, Uniqueness of generalized Schrödinger operators and applications, J. Funct. Anal., 105(1992), 187-231.

[13] M. RÖCKNER, T. S. ZHANG, Uniqueness of generalized Schrödinger operators and applications, II, J. Funct. Anal., 119(1994), 455-467.

[14] A. R. SCHEP, Weak Kato-inequality and positive semigroups, Math. Z., 190 (1985), 305-314.

[15] B. Simon, An abstract Kato's inequality for generators of positivity preserving semigroups, Indiana Univ. Math. J., 26 (1977), 1067-1073.

[16] N. WiELENS, On the essential self-adjointness of generalized Schrödinger operators, J. Funct. Anal., 61(1985), 98-115.

[17] L. M. WU, Uniqueness of Schrödinger operator restricted to a domain, J. Funct. Anal., 153(1998), 276-319.

[18] L. M. Wu, Uniqueness of Nelson's diffusions, Probab. Theory Relat. Fields, 114(1999), 549-585.

[19] L. M. WU, Y. ZHANG, A new topological approach for uniqueness of operators on $L^{\infty}$ and $L^{1}$ uniqueness of Fokker-Planck equations, J. Funct. Anal., 241(2006), 557-610. 